\title{
Improved K-Means Clustering Algorithm Based on KD-Tree Approach
}

\author{
Manish Bhardwaj' and Dattatraya Adane ${ }^{2}$ \\ ${ }^{1}$ Ph.D. Scholar, Computer Science and Engineering Department, Shri Ramdeobaba \\ College of Engineering and Management, Nagpur (MS), India. \\ ${ }^{2}$ Department of Information Technology, Shri Ramdeobaba College of Engineeing \\ and Management, Nagpur (MS), India.
}

\section{ABSTRACT}

The Cluster Analysis is a vast area of application such as security, Image recognition, scientific investigation, business intelligence, biology, and web search. K-Means clustering algorithm is not performing well with huge data sets in terms of Execution time. To overcome this, A Parallel Approach is used to implement the K-Means algorithm using OpenMP API with the KD-Tree approach to provide dynamic load balancing, optimized execution time, and maintaining accuracy. The experiments are performed on handwritten digits and Bagofword data sets by using a system with multi-core. After the analysis of the Sequential approach and Parallel approach of implementation of K-Means, it is observed that the parallel approach outperforms with similar accuracy utilizing the computing resources available with the multi-core systems.

KEY WORDS: K-MEANS, CLUSTERING ANALYSIS, OPENMP, PARALLEL APPROACH.

\section{INTRODUCTION}

A Clustering is the process of grouping similar data sets objects into clusters. The objects they are similar belong to the same cluster and the object they are dissimilar belong to other clusters. This concept of different applications in many areas such as biology, web, business, and security, etc. K-Means is a popular clustering technique. In the $\mathrm{K}$-Means clustering parameter $\mathrm{k}$, which is defined by the user is used to calculate the centroid. It is based on an iterative process that filters the result. If the calculated cluster point becomes the convergence then they are considered as the final result. In this process, the nearest neighbor search operation is used [Jiawei Han, 2012].

\section{ARTICLE INFORMATION}

*Corresponding Author: manish.bhardwaj1sept@gmail.com Received 15th Oct 2020 Accepted after revision 23rd Dec 2020 Print ISSN: 0974-6455 Online ISSN: 2321-4007 CODEN: BBRCBA

Thomson Reuters ISI Web of Science Clarivate Analytics USA and Crossref Indexed Journal

\section{Clarivate
Analytics}

NAAS Journal Score 2020 (4.31)

A Society of Science and Nature Publication,

Bhopal India 2020. All rights reserved.

Online Contents Available at: http//www.bbrc.in/

Doi: http://dx.doi.org/10.21786/bbrc/13.14/38
Different Clustering methods are used and can be classified as Partitioning methods, Hierarchical methods, Density-based methods, and Grid-based methods. $\mathrm{K}-$ Means Clustering is a Partitioning based method.

Parallel processing is used to solve a problem to reduce the time requirement of it. In Task partitioning, decomposition of solving procedure, and distribute them among the number of processors available and/or Data partitioning technique, data is divide and distributed among available processors and processor work with data in parallel. KD-Tree is a type of binary search tree, which is used for dynamic partitioning and overcome the problem associated with a static one. KD-Tree is constructed in parallel and shows better scalability. OpenMP API is useful in a shared memory environment, it take the advantage of multiple cores available in the system. The task is the process by multiple threads in a synchronous manner and the load is distributed among all threads. The Numbers of threads are created according to the number of processing elements present in a system. Independent blocks of code are required to find out and 
after the appropriate directives are placed before it. These are the indicators to compile the presence of a region that can be executed in parallel.

Related Work: Giuseppe Di Fatta and David Pettinger proposed and implemented improved K-Means algorithms based on the Binary Search Tree data structure called KD-Trees. To overcome the problem associated with static partitioning, dynamic partitioning is used for parallel implementation in a heterogeneous environment and it shows better scalability as compared to parallel brute force K-Means. The parallel algorithm totally depends on how the data sets are partitioned. KD-Tree construction is done in parallel. At last, the load is distributed using a dynamic load balancing technique [Giuseppe Di Fatta, 2010].

Qing Liao et al. proposed two strategies. Distance Measure strategy is the first approach in which the value of $q$ is used to choose the value of Distance. If $q$ is less than or equal to 8.495 Euclidean distance is chosen, if q is greater than this value Manhattan distance is chosen. The initial centroids selection strategy is the second approach if the data object belongs to a high-density area, in this case, it will be chosen as a centroid. By using this better stability and accuracy can be achieved [Qing Liao, 2013].

Mohammed Baydoun et al. implemented the K-Means algorithm in parallel by using OpenMP, Cilk Plus, MPI, and CUDA environment. OpenMP and Cilk Plus using a multithreaded programming model. In the CUDA environment, multiple GPUs are used, first threads are used to take care of the pattern and each thread maintained local memory to store the sample data which is used to choose the centroid. After analysis, they concluded that with small size data sets the OpenMP is performed better than all other but once the data size increase in that case with large data sets CUDA implementation shows better speedup [Mohammed Baydoun, 2016]. XiajKang Li et al. proposed the K-Means clustering method that is based on density. The mean shift algorithm is used to get local density maxima as initial cluster centers in place of computing the local density of each point cost 0 (n2) time. The mean shift algorithm is an iterative process to detect local maxima of the data set. The benefit of this approach is to choose parallel $\mathrm{K}$-appropriate local density maxima as initial cluster centers to cluster the data set in parallel [XiajKang Li, 2014].

S.N Trirumala rao et al. Proposed the parallel K-Means algorithm by using OpenMP API and mmape() function, to utilize the system page which improved the $\mathrm{I} / 0$ operation. First the data set is partition into the different block and one block at a time is cached into memory. In OpenMP API multiple threads are processing the data in parallel in a synchronous manner. This is the key factor for better I/O and speedup [ S. N Tirumala Rao, 2009]. Yufang Zhang et al. used a Master and slave technique to implement the K-Means in parallel. The master code is available in the host system. Its function is to distribute the data sets among multiple slaves and collect back the clustering result from them. The Load of the slaves is dynamically balanced. They proved that master and slave techniques show better efficiency [Yufang Zhang , 2006]. Juby Mathew et al. proposed the K-Means algorithm based on the concept of Firefly. The concept is based on the behavior of Firefly, how they are attracted to the light intense Firefly, in the same manner, the optimal cluster centroid can be obtained, and by using this optimal centroid the K-Means algorithms find more accurate clusters [Juby Mathew, 2015].

Improved Parallel Approach For K-Means Clustering: In Parallel approach for implementation of K-Means clustering, OpenMP construct play important role. It is working in the concept of Fork-Join. Where the task is divided and performs concurrently with the help of multiple threads, with proper synchronization and results are combine to generate overall result. First specific number of $\mathrm{K}$ is selected after that initial points are chosen by number of threads, each of the thread execute the Perform_clustering ( ). All threads are perform the operation in proper synchronization and cluster wise totals and cluster wise count is calculated. Once new Cluster points are calculated, they are compare with the previous cluster points, if both of the cluster points are same it is display as the final cluster points, Otherwise new cluster point is set as a currents cluster point and same operation of calculation of cluster points perform again till the convergence not meet.

Step 0. Select a suitable number of clusters, k.

Step 1. Select k initial cluster points.

Step 2. Choose the number of threads (let say 4 threads).

Step 3. By using section construct parallel section starts Perform_clustering ( );

Step 4. Apply the appropriate barrier for different threads.

Step 5. Calculate and use cluster wise count and Cluster wise total by using KD-Tree to dynamically balance the load among threads.

Step 6. Calculate again cluster points. Compare this cluster points with previous cluster points. If both the cluster points are existing cluster point is the same in this case display result otherwise again calculate the cluster point and repeat the previous condition.

Step7. Display Converge cluster points as a result.

Section constructs divide the task among threads. The Barrier construct is used to provide proper synchronization. Operations are performing in parallel with the help of Parallel construct with the proper declaration of shared and private variables [D. S. Bhupal Naik, 2013].

Experimental Analysis: In order to evaluate K-Means 
clustering method. It is implemented on system with Intel i3 CPU M380 processor with 4 GB RAM and 320 GB Hard Disk and Intel Pentium 4 processor with 1 GB RAM and 80 GB Hard Disk. The system is loaded with Ubuntu 18.04 and Ubuntu 16.04 operating system respectively. GCC compiler is used to execute the Parallel and Sequential implementation of Programs. The UCI Machine Learning Data Sets Repository and MNIST Database of Handwritten Digits are chosen and data repository. The K-Means Clustering method has analyzed on 2 Data Sets. Handwritten Digits and Bagofword. The analysis is performing on the basis of their sequential execution and parallel execution time requirement. Later the same is used for defining speedup and comparison between the sequential and parallel implementation of programs.

Table 1. Performance Analysis of Handwritten Digits data with Number of Objects $=2000$

\begin{tabular}{|l|c|c|c|}
\hline $\begin{array}{l}\text { Number } \\
\text { of Cluster }\end{array}$ & $\begin{array}{c}\text { PTime } \\
\text { (in seconds) }\end{array}$ & $\begin{array}{c}\text { STime } \\
\text { (in seconds) }\end{array}$ & Speedup \\
\hline 2 & 0.3606 & 0.9347 & 2.5806 \\
\hline 4 & 0.5391 & 1.4775 & 2.7406 \\
\hline 6 & 0.6526 & 1.8436 & 2.8250 \\
\hline 8 & 1.1439 & 3.2829 & 2.8699 \\
\hline 10 & 2.0694 & 6.0241 & 2.9079 \\
\hline
\end{tabular}

Table 2. Performance Analysis of Handwritten Digits data with Number of Objects $=70000$

\begin{tabular}{|l|c|c|c|}
\hline $\begin{array}{l}\text { Number } \\
\text { of Cluster }\end{array}$ & $\begin{array}{c}\text { PTime } \\
\text { (in seconds) }\end{array}$ & $\begin{array}{c}\text { STime } \\
\text { (in seconds) }\end{array}$ & Speedup \\
\hline 2 & 12.3169 & 32.0211 & 2.5997 \\
\hline 4 & 21.5585 & 59.5629 & 2.7628 \\
\hline 6 & 33.6989 & 96.2007 & 2.8547 \\
\hline 8 & 48.0502 & 139.2176 & 2.8973 \\
\hline 10 & 111.7732 & 311.9214 & 2.7906 \\
\hline
\end{tabular}

Table 3. Performance Analysis of Bagofword data with Number of Objects $=3710423$

\begin{tabular}{|l|c|c|c|}
\hline $\begin{array}{l}\text { Number } \\
\text { of Cluster }\end{array}$ & $\begin{array}{c}\text { PTime } \\
\text { (in seconds) }\end{array}$ & $\begin{array}{c}\text { STime } \\
\text { (in seconds) }\end{array}$ & Speedup \\
\hline 2 & 0.1913 & 0.4001 & 2.0914 \\
\hline 3 & 0.2157 & 0.4788 & 2.2197 \\
\hline 4 & 0.2559 & 0.5996 & 2.3431 \\
\hline 5 & 0.2828 & 0.7237 & 2.5590 \\
\hline
\end{tabular}

The Table I show the time required of data set with 2000 objects in seconds for clustering. Its shows both sequential as well as parallel implementation time requirements. The Speedup achieved by parallel execution is $\sim 2.78$. The
Table 4. Performance Analysis of Bagofword data with Number of Objects $=69679430$

\begin{tabular}{|l|c|c|c|}
\hline $\begin{array}{l}\text { Number } \\
\text { of Cluster }\end{array}$ & $\begin{array}{c}\text { PTime } \\
\text { (in seconds) }\end{array}$ & $\begin{array}{c}\text { STime } \\
\text { (in seconds) }\end{array}$ & Speedup \\
\hline 2 & 3.5246 & 8.0604 & 2.2868 \\
\hline 3 & 3.9926 & 14.043 & 3.4847 \\
\hline 4 & 4.7307 & 14.7532 & 3.1186 \\
\hline 5 & 5.2536 & 16.9116 & 3.2190 \\
\hline
\end{tabular}

Figure 1: Performance Graph based on Table I

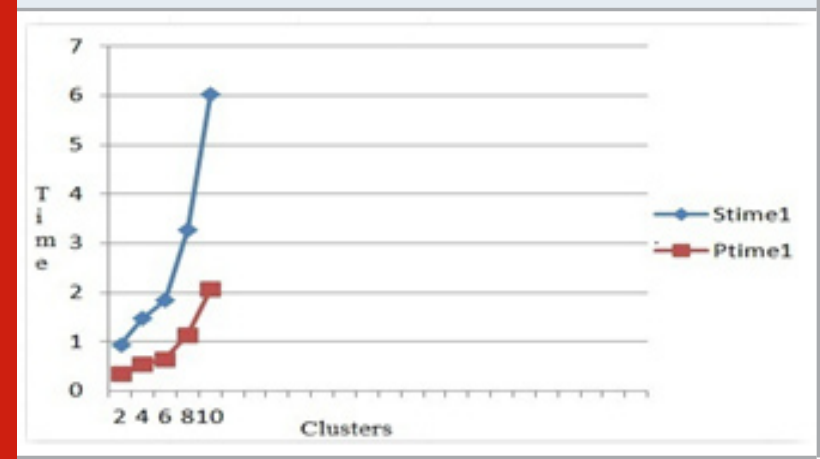

Figure 2: Performance Graph Based on Table II

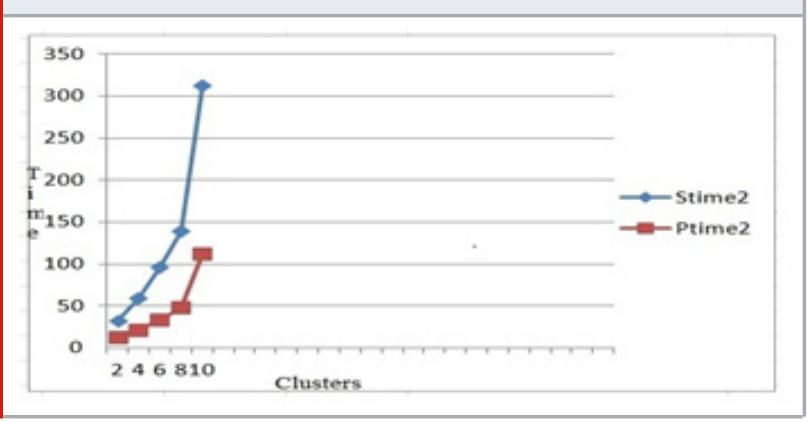

Table II shows the time required of data set with 70000 objects in seconds for clustering. Its shows both sequential as well as parallel implementation time requirements. The Speedup achieved by parallel execution is $\sim 2.78$. The Table III shows the time required of data set with 3710423 objects in seconds for clustering.

Figure 3: Performance Graph Based on Table III

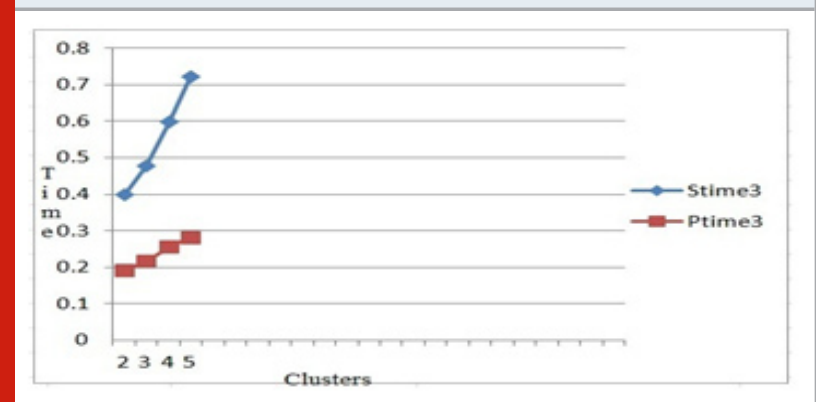


Figure 4: Performance Graph Based on Table IV

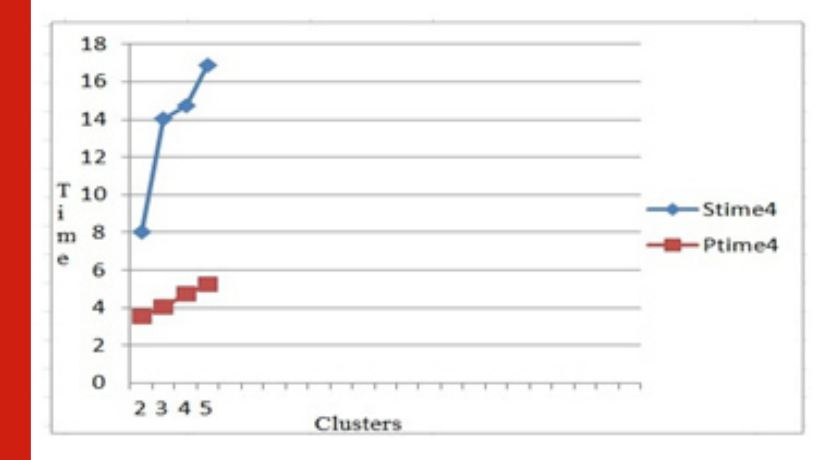

It is shows both sequential as well as parallel implementation time requirements. The Speedup achieved by parallel execution is $\sim 2.3$. The Table IV shows the time required of data set with 69679430 objects in seconds for clustering. It is shows both sequential as well as parallel implementation time requirements. The Speedup achieved by parallel execution is $\sim 3.02$.

\section{REFERENCES}

Barbara Chapman, Gabriele Jost and Ruud Vander Pas, Using OpenMP Portable Shared Memory Parallel Programming, The MIT Press, Cambridge Massachusetts London England, 2008. Chapter 1, pp 28-34.

D. S. Bhupal Naik, S. Deva Kumar and S.V. Ramakrishna,"Parallel Processing of Enhanced K-Means Using OpenMP" IEEE International Conference on Computational Intelligence and Computing Research, 2013, pp 1-5.

David Newman, Data set Bag of Words, UCI Machine Learning Repository, http://archive.ics.uci.edu/ml, University of California, School of Information and Computer Science, 2008.

Giuseppe Di Fatta and David Pettinger, "Dynamic Load Balancing in Parallel KD-Tree K-Means" 10th IEEE International Conference on Information Technology, 2010, pp 1-5.
Jeyhun Kavimov, Murat Ozbayoglu and Erdogan Dogdu, "K-Means performance Improvements with Centroid Calculation Heuristics both for Serial and Parallel Environment" IEEE International Congress on Big Data, 2015, pp 444-451.

Jiawei Han, Micheline Kamber and Jian Pei, Data Mining Concepts and Techniques, Morgan Kaufmann Publishers USA, 2012, Chapter 3, pp 443-454.

Mathew J and Vijayakumar R, "Enhancement of Parallel K-Means Algorithm" IEEE Sponsored 2nd International Conference on Innovation in Information Embedded and Communication System, 2015, pp 1-5.

Mohammed Baydoun, Mohammad Dawi and Hassan Ghaziri,“Enhanced Parallel Implementation of K-Means Clustering Algorithm" 3rd International conference on Advances in Computation Tools for Engineering Applications , 2016, pp 1-5.

Patrick Markey and Robert R. Levis, "Parallel K-Means++ for Multiple Shared- Memory Architectures" 45th International conference on Parallel Processing, 2016, pp 1-5.

Qing Liao, Fan Yang and Jingming Zhao, "An Improved Parallel K-Means Clustering Algorithm with Map Reduce" 15th IEEE International Conference on Communication Technology, 2013, pp 1-7.

S. N. Tirumala Rao, E.V. Prasad, and N.B. Venkatewarlu, "A Scalable K-Means Clustering Algorithm on MultiCore Architecture" International Conference on Methods and Models in Computer Science, 2009, pp 1-7.

XiajKang Li, Binbin Xu, Yinghua Zhou and GuangZhong Sun, "A Novel Density based Clustering Algorithm and Its Parallelization" 15th International conference on Parallel and Distributed computing, Application and Technologies, 2014, pp 1-5.

Yann Lecun, Corinna Cotes and Christopher J.C. Bugers, Data set Handwritten Digits, MNIST Database, 2016.

Yufang Zhang, Zhongyang Xiong, Jiaili Mao and Ling $\mathrm{Ou}$, "The Study of Parallel K-Means" 6th World Congress on Intelligent control and Automation, 2006, pp 58685871. 